\title{
Energy Aware Routing Protocol in MANET using Power Efficient Topology Control Method
}

\author{
Suchismita Rout \\ Department of Computer Sc. \\ and Engineering \\ National Institute of \\ Technology, Rourkela \\ Rourkela, India
}

\author{
Ashok Kumar Turuk \\ Department of Computer Sc. \\ and Engineering \\ National Institute of \\ Technology, Rourkela \\ Rourkela, India
}

\author{
Bibhudatta Sahoo \\ Department of Computer Sc. \\ and Engineering \\ National Institute of \\ Technology, Rourkela \\ Rourkela, India
}

\begin{abstract}
In MANET nodes are battery operated with dynamic network topology due to mobility of nodes. . Therefore energy efficiency is an important design consideration to extend the lifetime of networks. Topology of network plays an important role for energy conservation. This paper addresses how the topology of the network can be adjusted by controlling the transmission power. In this work the node in the farthest transmission range will take part in routing and the node that is geographically closer to the destination node is the candidate. Energy conservation is based on sleep based approaches. The energy is conserved by controlling a set of neighbor to which the node communicates. We have simulated our proposed scheme using Qualnet 4.5 simulator. Simulation results shows that proposed approach has a good energy conservation performance and also performs better in context of average end-to-end delay without much affecting the throughput.
\end{abstract}

\section{General Terms}

Mobile ad-hoc network

\section{Keywords}

Energy aware protocol, topology control, routing, farthest node, common node, sleep based approach.

\section{INTRODUCTION}

Mobile ad-hoc network (MANET) has an explosion of interest from consumers in recent years for its applications in mobile and personal communication [1]. The nodes configure themselves dynamically to establish network connectivity [2]. Since energy is the limiting factor for lifetime and connectivity in MANET, many research works have been progressed in this area to conserve energy. As servicing and replacing batteries may not be feasible for some applications, some power aware communications and computing has received considerable attention for extending network life time [3].

Different energy aware techniques have been proposed from several years. Topology control approach is one of these techniques. Topology of network is usually temporary due to mobility of nodes [4]. For energy efficient routing an appropriate transmission power of data packets at each node is decided. Fixed transmit power approach doesn't give guaranty of finding neighbor of a node $[5,6]$. Without topology control mechanism each node transmits their packets with maximum power.
Power-efficient topology control method is to give the reasonable transmission power of each node so that a moderate connectivity is maintained in networks. It also reduces the total energy consumption for per-packet transmission. A node selects a closest neighbor list coming in its transmission range. The transmission range is properly adjusted using topology control mechanism. Then the farthest node coming in its range will take part in next hop for routing. This node should be geographically closer to the destination node. Each node continuously considers which neighbor it can exclude from direct communication to conserve power. Idle mode nodes are put into sleep mode for increasing energy efficiency. Topology control plays an important role in maintaining network connectivity and efficiently utilizes node's energy for longer network lifetime [7].

In our paper, we propose a distributed protocol, named Distance-based Sleep Scheduling (DBSS) protocol, to deal with topology control problem at network layer and also help to reduce total energy consumption of network to maximize network lifetime.

Rest of the literatures has been arranged as follows. Section 2 focuses on related work. Mathematical model for MANET is described in section 3. The mathematical model represents the routing in MANET by considering both the distance and residual energy of the node. Section 4 discusses the effect of topology on energy aware routing in MANET. Our proposed approach has been discussed in section 5 and its simulation behavior is compared with existing LFTC protocol in section 6. It is followed by conclusions and acknowledgements in section 7 and 8 respectively. Finally references are listed in section 9 .

\section{RELATED WORK}

Resource limitation, mobility of hosts and changing of wireless link make it difficult for MANET to manage for all quality of services. In spite of all these difficulties MANET is a good candidate for various military and civil applications. A lot of research works have been progressed in the area for energy conservation in MANET. For efficient operations of network with the changing topology with the node mobility generates higher control message overhead. Methods to reduce energy consumption include: 
- Considering residual battery energy while selecting the route.

- Reducing the communication overhead of control messages.

- Efficient route reconfiguration mechanisms (effect in topology changes).

Total energy consumption is divided into two parts. The former is $\mathrm{E}_{\text {path-discovery }}$ and later $\mathrm{E}_{\text {packet-transmission. }}$. $\mathrm{E}_{\text {path-discovery }}$ is directly proportional to number of control packets. A node in packet transmission consumes energy in four states. Those are as follows: (a) transmit, (b) receive, (c) idle and (d) sleep. Highest power is consumed in transmit mode and least in sleep mode. Node does not do any useful work in idle mode. In idle mode the power consumption is as high as in receive mode. So in this mode energy is wasted unnecessarily. The principal sources of energy waste in MAC assume collision, message overhearing (receiving packets addressed for other nodes), and control packets overhead and idle listening.

$$
\begin{aligned}
& E_{\text {total }}=E_{\text {path-discovery }}+E_{\text {packet-transmisson }} \\
& E_{\text {path-discovery }} \text { acontrol - packets }
\end{aligned}
$$

$$
E_{\text {packet-transmissin }}=E_{\text {idle }}+E_{\text {active }}+E_{\text {sleep }}+E_{\text {transient }}
$$

$$
E_{\text {active }}=E_{\text {recv }}+E_{\text {transmit }}
$$

$$
E_{\text {sleep } \cong o}
$$

Traditional routing protocol find the route depending upon the shortest path mechanism which is not appropriate for MANET, as a very small set of nodes are overuse rapidly in favor of others may cause network partitioning. To increase node and network life time the route is established by taking the lightly loaded nodes with sufficient power resources [8]. For longer network life and minimize energy consumption the basic solution to this problem is organized is three parts.

- Power Management Approach

- Power Control Approach

- Topology Control Approach

Energy conservation is achieved not by taking any solutions alone; it is possible by taking the combination of two or three techniques simultaneously. There are many researches carried out in order to reduce energy consumption in MANET. Some of them are explained as follows.

\subsection{DEAR Protocol}

DEAR stands for Device and Energy Aware Routing. DEAR protocol explains the use of device awareness to enhance energy efficiency in the routing. A node is assumed to be device aware if it is assumed to be powered by two states: internal battery power and external power source [9]. It assumes the cost of a node powered by external source is zero. The packets can be redirected to the powered node for power saving operations. An externally powered node has rich resource of power. It is capable of increasing its transmission power to a higher level so that it is easily reachable to any desired node in network in one hop distance. DEAR provides power saving by eliminating a number of hops which increases system life time, also average delay in packet receiving is minimized.

\subsection{SPAN Protocol}

Nodes in idle mode unnecessarily consume energy. To save energy it is better to put those nodes in sleep state without hampering network connectivity. For taking this decision a master node is selected. The SPAN protocol [10] employs a distributed approach to select a master node. The rule says that if two of its neighbors cannot reach each other either directly or via one or two masters, it should become a master. This rule does not yield the minimum number of master nodes. It provides robust connectivity with substantial energy savings. However, the master nodes are easily overloaded. To minimize this, the master node at any time withdraws as a master. It gives its neighbor node a chance to become a master if it satisfies master eligibility criteria.

\subsection{XTC Protocol}

The XTC topology control algorithm [11] works without either location or directional information. The algorithm has three phases. In first one each node broadcast at maximum power. Then it ranks its entire neighbor depending upon its link quality to it. The link quality could be the Euclidean distance, signal attenuation or packet arrival rate depending on various situations. In second steps each node transmits its ranking results to neighboring nodes. In the final one, each node examines the ranking results of its neighbors. Depending upon this result it select neighbor to be linked directly. The XTC algorithm follows both symmetry and connectivity feature of topology control. It runs faster as compare to other algorithms.

\subsection{LFTC Protocol}

The LFTC protocol [12] constructs power-efficient network topology. It also avoids any potential collision due to hidden terminal problem. It works in two phases: link determination phase and interference announcement phase. In first phase each node broadcasts hello message with vicinity table. This table is attained by every other node in network. Each node adjusts its transmission power $\mathrm{P}_{\text {data }}$ to communicate with all its direct neighbors. The set of its direct neighbors is called direct communication set (DCS). The second phase avoids data collision due to hidden terminal problem. This is done by taking appropriate power for $\mathrm{P}_{\text {data }}$ and $\mathrm{P}_{\text {contro }}$. This results in two simultaneous data transmission in networks without any interference. The LFTC protocol has smaller hop count, low control packet overhead and low ratio of collision as compare to XTC. 
In this paper we put more focus on topology control approach for minimizing energy consumption in networks. Before that we present the mathematical model for MANET. This model represents the behavioral model of MANET and show the effect of distance between two nodes and residual energy of the node on node behavior.

\section{MATHEMATICAL MODEL FOR MANET:}

In MANET, a network can be represented by a weighted graph $\mathrm{G}=(\mathrm{V}, \mathrm{E})$, where $\mathrm{V}$ is the set of nodes and $\mathrm{E}$ is the set of links between two nodes. All the nodes of network are in mobile state. The position, rate and direction of motion of node $\mathrm{V}_{\mathrm{i}}$ at time $\mathrm{t}$ can be represented as $\left.\left(\mathrm{x}_{\mathrm{i}}(\mathrm{t}), \mathrm{y}_{\mathrm{i}}(\mathrm{t})\right), \mathrm{s}_{\mathrm{i}}(\mathrm{t}), \theta_{\mathrm{i}}(\mathrm{t})\right)$ respectively. So the motion model is represented as follows [13]:

$$
\left\{\begin{array}{l}
x_{i}(t)=x_{i}(t-1)+s_{i}(t) \times \cos \left(\theta_{i}(t)\right) \\
y_{i}(t)=y_{i}(t-1)+s_{i}(t) \times \sin \left(\theta_{i}(t)\right)
\end{array}\right.
$$

The distance between two nodes in MANET is represented as follows:

$$
d_{i j}=\sqrt{\left(x_{j}(t)-x_{i}(t)\right)^{2}+\left(y_{j}(t)-y_{i}(t)\right)^{2}}
$$

The nodes are deployed in free space to establish network connectivity. Two nodes are connected to each other if they are in transmission range of each other. The link between two nodes can be possible if the signals from the transmitter can be received correctly by other node. The distance $\mathrm{R}$ is the effective distance between two nodes when routing link between two nodes sets apart. So the relation between communication link and distance between two nodes are summarized below:

$$
d_{i j}(t) \leq R \Leftrightarrow\left(v_{i}, v_{j}\right) \in E
$$

If $\left(\mathrm{v}_{\mathrm{i}}, \mathrm{v}_{\mathrm{j}}\right)$ distance is within the range of effective distance then $\mathrm{v}_{\mathrm{i}}$ and $\mathrm{v}_{\mathrm{j}}$ are called as adjacent nodes. An $\mathrm{N} \times \mathrm{N}$ adjacency matrix can be constituted by MANET of $\mathrm{N}$ nodes; the elements should satisfy the following relations:

$$
a_{i j}=\left\{\begin{array}{l}
1,\left(v_{i}, v_{j}\right) \in E \\
0, \text { otherwise }
\end{array}\right.
$$

A $\mathrm{N} \times \mathrm{N}$ weight matrix $\mathrm{W}=\left(\mathrm{w}_{\mathrm{ij}}\right)_{\mathrm{N} \times \mathrm{N}}$ consists of weight matrix of a network of $\mathrm{N}$ number of nodes. In routing distance between two nodes are considered as the weight of links. Elements of weight matrix can be rewritten as:

$$
w_{i j}=\left\{\begin{array}{l}
d_{i j},\left(v_{i}, v_{j}\right) \in E \\
\infty, \text { otherwise }
\end{array}\right.
$$

In MANET the topology of deployment is not constant as the nodes are mobile in nature. Both of these adjacency matrix and weight matrix are time varying matrices. In network each node has a unique identifier. $\mathrm{S}$ is the identifier of source node and $\mathrm{D}$ is for destination node, all the other nodes except source node and destination node are called intermediate node. Then the optimization model of shortest routing can be represented as follows:

$$
\begin{aligned}
& \min \sum_{\left(v_{i}, v_{j}\right) \in E} w_{i j} x_{i j}
\end{aligned}
$$

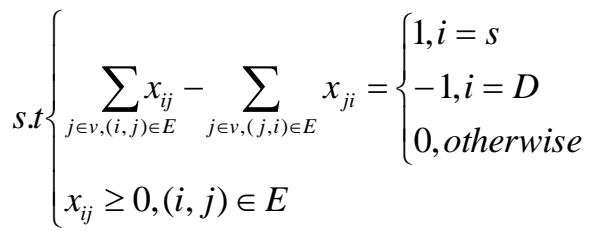

We can get $\mathrm{N} \times \mathrm{N}$ matrix in which the link between $\mathrm{v}_{\mathrm{i}}$ and $\mathrm{v}_{\mathrm{j}}$ is chosen as shortest routing path if $\mathrm{x}_{\mathrm{ij}}=1$.

In routing selection the length of path is considered as one factor. But by using a single path repeatedly the energy of the nodes of that path is gradually decreasing earlier than the other nodes of network. That node is called as bottleneck node. For optimization model we take both the residual energy and distance between two nodes as two factors.

As we have mentioned earlier that a network is represented as $\mathrm{N}=(\mathrm{V}, \mathrm{E})$, where $\mathrm{V}$ is the set of nodes and $\mathrm{E}$ is the set of communication links. Each node has a unique identifier, $\mathrm{S}$ is the identifier of source node and D is for destination node. Each edge is carrying a non-negative capacity function cap(e). A network flow from source to destination is a mapping $f$ that maps each edge e a value $f(e)$ such that following conditions are satisfied.

1. Capacity constraint: $0<=\mathrm{f}(\mathrm{e})<=\mathrm{cap}(\mathrm{e})$, that is no flow exceeds the feasible capacity. Feasible capacity in network terminology is the minimum residual energy of two nodes that is required to constitute the edge. The capacity function can be further represented as follows :

$$
c\left(v_{i}, v_{j}\right)=\min \left(E\left(v_{i}\right), E\left(v_{j}\right)\right)
$$

2. Flow conservation: For each node except source and destination the following holds: The sum of the $\mathrm{f}(\mathrm{e})$ over all edges e incident to $\mathrm{v}$ is equal to the sum of the $\mathrm{f}(\mathrm{g})$ over all edges $\mathrm{g}$ leaving $\mathrm{v}$.

For all the intermediate nodes the value of flow entering is equal to the flow leaving from the node. But for the source node $\mathrm{S}$, all the flows are leaving. Similar for the destination node the flow are entering into the node. The traffic flow $V_{f}$ of a certain routing is exactly residual energy of the bottleneck node in networks. It should be equal to the $\mathrm{f}(\mathrm{g})$ that outflow for the source node and inflow $\mathrm{f}(\mathrm{g})$ for the destination node. For intermediate node the outflow $\mathrm{f}(\mathrm{g})$ is equal to the inflow $\mathrm{f}(\mathrm{e})$. So the residual energy model can be described as follows: 


$$
\begin{aligned}
& \min v_{f} \\
& s . t\left\{\begin{array}{l}
\sum_{j \in v,(i, j) \in E} x_{i j} f_{i j}-\sum_{j \in v,(j, i) \in E} x_{j i} f_{j i}=\left\{\begin{array}{l}
f(g), i=s \\
f(e), i=D \\
0, \text { otherwise }
\end{array}\right. \\
\sum_{j \in v,(i, j) \in E} x_{i j}-\sum_{j \in v,(j, i) \in E} x_{j i}=\left\{\begin{array}{l}
1, i=s \\
-1, i=D \\
0, \text { otherwise }
\end{array}\right. \\
0 \leq f\left(v_{i}, v_{j}\right) \leq c\left(v_{i}, v_{j}\right) \\
x_{i j} \geq 0,(i, j) \in E
\end{array}\right.
\end{aligned}
$$

$V_{f}$ is same as the bottleneck residual energy in selected routing. In routing by utilizing a single path its energy decreasing rapidly, to improve the performance of network it is combined with shortest path routing. The above equation is rewritten as follows:

$$
\begin{aligned}
& \max \sum_{j=1,(i, j) \in E}^{N} x_{i j} w_{i j} f_{i j} \\
& s . t\left\{\begin{array}{l}
\sum_{j \in v,(i, j) \in E} x_{i j} f_{i j}-\sum_{j \in v,(j, i) \in E} x_{j i} f_{j i}=\left\{\begin{array}{l}
f(g), i=s \\
f(e), i=D \\
0, \text { otherwise }
\end{array}\right. \\
\sum_{j \in v,(i, j) \in E} x_{i j}-\sum_{j \in v,(j, i) \in E} x_{j i}=\left\{\begin{array}{l}
1, i=s \\
-1, i=D \\
0, \text { otherwise }
\end{array}\right. \\
0 \leq f\left(v_{i}, v_{j}\right) \leq c\left(v_{i}, v_{j}\right) \\
x_{i j} \geq 0,(i, j) \in E
\end{array}\right.
\end{aligned}
$$

In the above model we set up routing mechanism with careful consideration of residual energy and distance between two nodes, for energy conservation. In this paper the node in the farthest transmission range of a node is chosen as next hop node for transferring packet. This node is geographical closer to the destination node. So that the resulting path has less number of hop counts. So the total energy consumption of the network is less, which increases network efficiency.

\section{TOPOLOGY CONTROL}

The topology control is an effective technique for power saving. Mobility of wireless nodes makes the topology of network changes temporary. It is affected by many uncontrollable factors like node mobility, weather conditions, environmental interference and obstacles and some controllable factors like transmission power, antenna direction and duty-cycle scheduling. Topology of network is considered as graph with its nodes as vertices and communication links between node pairs as edges. The edge set is large possible one if communication is established by node's maximum transmission power. In dense network too many links leads to high energy consumption. The primary target of topology control is to replace long distance communication with small energy efficient hops. Dense network ensures tight connectivity and high interference. In sparse network connectivity between nodes is being a question. So there is a tradeoff between network connectivity and sparseness [11].

Network topology can enhance network throughput because of two benefits. First the interference is reduced if transmission radii of nodes are reduced to near one. Second more data transmission is carried out simultaneously in the neighborhood of a node. A bad network topology has many adverse effects such as low capacity, high end-to-end delay and weak robustness to node failure. Where as a good network topology minimize energy consumption and end to end delay without much affecting the throughput [14].

\section{PROPOSED APPROACH}

The proposed method utilizes the transmission power of node to control network the topology. It also follows sleep based approach for nodes in idle mode. That results in energy conservation in network with proper connectivity. The protocol environmental assumptions are described as follows:

1. We are considering two dimensional homogeneous networks where all the nodes have limited battery power and similar capabilities. Each node has its own ID and can communicate to other nodes through Omni-directional antenna.

2. Nodes are aware of their exact co-ordinates and the co-ordinates of the destination to which the source node needs to send the data.

3. The signals from neighbor nodes can be received accurately and the received signal power can be measured with the help of radio interface at each node.

The initial topology is $\mathrm{G}=(\mathrm{V}, \mathrm{E})$ before power efficient topology control is applied. Let $\mathrm{P}_{\mathrm{uv}}$ denotes the minimum power required for node $\mathrm{u}$ to communicate directly to node $\mathrm{v}$. By referring the model presented in [15] for node $u$ to determine the power $\mathrm{P}_{\mathrm{uv}}$ where $\mathrm{v}$ sends a message to $\mathrm{u}$, and v's maximum transmission power $\mathrm{P}_{\max }$ is known to $\mathrm{u}$. Suppose that $u$ receives the message with power $P_{r}$, and $P_{\min }$ denotes a node's smallest possible receiving power. Then

$$
P_{u v}=\frac{P_{\max } \cdot P_{\min }}{P_{r}}
$$

This is based on Equation (5)

$$
P_{r}=P_{t}\left(\frac{\lambda}{4 \pi d}\right)^{n} \cdot g_{t} \cdot g_{r}
$$

Where, $\mathrm{P}_{\mathrm{t}}$ and $\mathrm{P}_{\mathrm{r}}$ denote the signal power at transmitting and receiving antenna, respectively, $\lambda$ denotes the carrier wavelength, $d$ denotes the distance between the sender and the receiver, and $g_{t}$ and $g_{r}$ denote the antenna gains at the sender and receiver, respectively. The energy cost for sending packet from $\mathrm{u}$ to $\mathrm{v}$ is denoted as $\mathrm{C}\left(\mathrm{P}_{\mathrm{uv}}\right)$ where $\mathrm{C}\left(\mathrm{P}_{\mathrm{uv}}\right)=\mathrm{C}\left(\mathrm{P}_{\mathrm{vu}}\right)$ as medium is symmetric. All nodes have common maximum 
transmission power denoted as $\mathrm{P}_{\max }$, the nodes can change their transmission power below $\mathrm{P}_{\max }$.

Our protocol consists of two phases: the first phase is the link determination phase, and the second one is sleep scheduling phase. In link determination phase each node, say $u$, independently selects the set of its neighbors according to a power efficient topology control method. Those chosen next hop nodes who are coming under transmission range of node $\mathrm{u}$ will coming under Direct communication set of node $\mathrm{u}$, called DCS $(\mathrm{u})$. The data packet transmission power of node $\mathrm{u}$, $\mathrm{P}_{\text {data }}(\mathrm{u})$ is determined at the end of link determination phase which is the minimum power by which a node can communicate to all of its direct neighbors of DCS(u). In sleep scheduling phase a node determines its farthest node but geographically closer to the destination node to be chosen as next hop neighbor for packet transferring [7], and make other nodes to be in sleep mode instead of putting them in idle mode so that energy can be saved.

\subsection{Link Determination Phase}

In the first phase a node randomly broadcast the hello message once using the power $P_{\max }$. It is the constant transmission power of each node. Node computes $\mathrm{P}_{\mathrm{uv}}$ and $\mathrm{C}\left(\mathrm{P}_{\mathrm{uv}}\right)$ after listening to hello message. Each hello message contains the specific data structure called vicinity table as given in Table 1. There are six fields in the vicinity table. The first field, sender-ID records the node's ID which sends the hello message. The second field appends the sender-location information in the table. The field direct-comm-cost stores $\mathrm{C}\left(\mathrm{P}_{\mathrm{uv}}\right)$ which is required cost when a node $\mathrm{u}$ directly communicates with node v. The min-comm-cost stores the minimum cost of communication energy from node $u$ to $v$. The common-node field contains the common energy efficient node between node $\mathrm{u}$ and node $\mathrm{v}$. This node makes cost of communication less than direct-comm-cost. This field can be null or more than one node entry depending upon the communicating path between two nodes. This field is null if it is direct (one-hop) otherwise contain an entry if indirect (multi-hop). The last field link-type is marked as "d" or "i". Where "d" indicates whether the node is its direct or one-hop neighbor otherwise it is marked as " $\mathrm{i}$ " if it is its indirect or multi-hop neighbor.

The content of vicinity table of each node is empty at the beginning. A node sends the hello message with power $\mathrm{P}_{\max }$. It appends its ID and location information in the field of vicinity

Table 1: Vicinity Table

\begin{tabular}{|c|c|c|c|c|c|}
\hline $\begin{array}{c}\text { Sender- } \\
\text { ID }\end{array}$ & $\begin{array}{c}\text { Location- } \\
\text { Info }\end{array}$ & $\begin{array}{c}\text { Min- } \\
\text { comm- } \\
\text { cost }\end{array}$ & $\begin{array}{c}\text { Common- } \\
\text { node }\end{array}$ & $\begin{array}{c}\text { Direct- } \\
\text { comm- } \\
\text { cost }\end{array}$ & $\begin{array}{c}\text { Link- } \\
\text { type }\end{array}$ \\
\hline
\end{tabular}

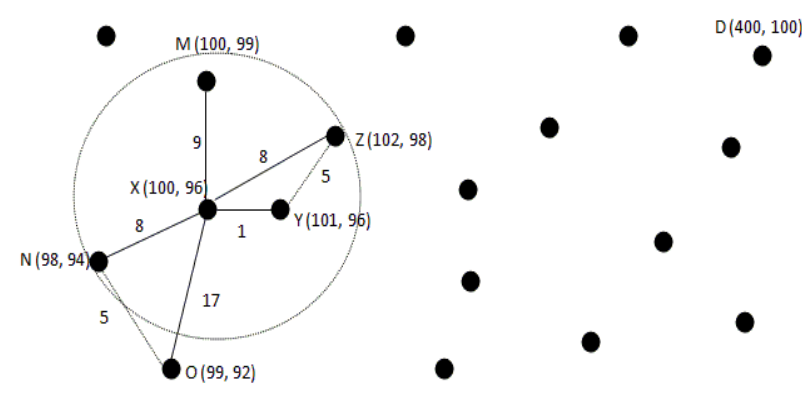

Figure 1: A Network structure

table. The other fields are remaining empty as given in Table 2. The receiver collects the information from the vicinity table. Depending upon the information it updates the fields of the vicinity table as given in Table 3 .

Table 2: Vicinity Table present in hello msg of node $Y$

\begin{tabular}{|c|c|c|c|c|c|}
\hline $\begin{array}{c}\text { Sender- } \\
\text { ID }\end{array}$ & $\begin{array}{c}\text { Location- } \\
\text { Info }\end{array}$ & $\begin{array}{c}\text { Min- } \\
\text { comm- } \\
\text { cost }\end{array}$ & $\begin{array}{c}\text { Common- } \\
\text { node }\end{array}$ & $\begin{array}{c}\text { Direct- } \\
\text { comm- } \\
\text { cost }\end{array}$ & $\begin{array}{c}\text { Link- } \\
\text { type }\end{array}$ \\
\hline $\mathrm{Y}$ & 101,96 & - & - & - & - \\
\hline
\end{tabular}

The distance between two point $\mathrm{X}$ and $\mathrm{Y}$ is calculated using Equation 6.

$$
d=\sqrt{\left(X_{2}-X_{1}\right)^{2}+\left(Y_{2}-Y_{1}\right)^{2}}
$$

Where $\left(\mathrm{X}_{1}, \mathrm{Y}_{1}\right)$ is the co-ordinate of node $\mathrm{X}$ and $\left(\mathrm{X}_{2}, \mathrm{Y}_{2}\right)$ is the co-ordinate of node $\mathrm{Y}$.

Table 3: Vicinity Table of node $X$

\begin{tabular}{|c|c|c|c|c|c|}
\hline $\begin{array}{c}\text { Sender- } \\
\text { ID }\end{array}$ & $\begin{array}{c}\text { Location- } \\
\text { Info }\end{array}$ & $\begin{array}{c}\text { Min- } \\
\text { comm- } \\
\text { cost }\end{array}$ & $\begin{array}{c}\text { Common- } \\
\text { node }\end{array}$ & $\begin{array}{c}\text { Direct- } \\
\text { comm- } \\
\text { cost }\end{array}$ & $\begin{array}{c}\text { Link- } \\
\text { type }\end{array}$ \\
\hline $\mathrm{Y}$ & 101,96 & 01 & - & 01 & $\mathrm{D}$ \\
\hline
\end{tabular}

For example in Figure 1, node $\mathrm{X}$ has received the hello messages from its neighbor nodes. These nodes are coming in its transmission power $\mathrm{P}_{\max }$. Then it establishes the vicinity table accordingly as given in Table 4 .

Table 4: Vicinity Table of node $X$

\begin{tabular}{|c|c|c|c|c|c|}
\hline $\begin{array}{c}\text { Sender- } \\
\text { ID }\end{array}$ & $\begin{array}{c}\text { Location- } \\
\text { Info }\end{array}$ & $\begin{array}{c}\text { Min- } \\
\text { comm- } \\
\text { cost }\end{array}$ & $\begin{array}{c}\text { Common- } \\
\text { node }\end{array}$ & $\begin{array}{c}\text { Direct- } \\
\text { comm- } \\
\text { cost }\end{array}$ & $\begin{array}{c}\text { Link- } \\
\text { type }\end{array}$ \\
\hline $\mathrm{Y}$ & 101,96 & 01 & - & 01 & $\mathrm{D}$ \\
\hline $\mathrm{Z}$ & 102,98 & 08 & - & 08 & $\mathrm{D}$ \\
\hline
\end{tabular}




\begin{tabular}{|c|c|c|c|c|c|}
\hline $\mathrm{M}$ & 100,99 & 09 & - & 09 & $\mathrm{D}$ \\
\hline $\mathrm{N}$ & 98,94 & 08 & - & 08 & $\mathrm{D}$ \\
\hline $\mathrm{O}$ & 99,92 & 17 & - & 17 & $\mathrm{D}$ \\
\hline
\end{tabular}

Each node has initial information about its neighbor nodes. The collected information is used by node to find any common node. In wireless transmission the minimum energy required to successfully transmit a data packet from source to destination increases with the distance between them [16]. By using the common node the cost of communication is decreasing.

Common node finding algorithm ( ):

1. Given: the co-ordinates of 3 points $X\left(X_{1}, Y_{1}\right), Y\left(X_{2}\right.$, $\left.\mathrm{Y}_{2}\right)$ and $\mathrm{Z}\left(\mathrm{X}_{3}, \mathrm{Y}_{3}\right)$.

2. Check for co-linearity of $\mathrm{X}, \mathrm{Y}$ and $\mathrm{Z}$.

If $\left[\left(\mathrm{X}_{2}-\mathrm{X}_{1}\right) *\left(\mathrm{Y}_{3^{-}} \mathrm{Y}_{1}\right)\right]==\left[\left(\mathrm{X}_{3^{-}} \mathrm{X}_{1}\right) *\left(\mathrm{Y}_{2}-\mathrm{Y}_{1}\right)\right]$

Z.

$\mathrm{X}, \mathrm{Y}$ and $\mathrm{Z}$ are collinear and $\mathrm{Y}$ is in between $\mathrm{X}$ and

3. Y's location lies in between $\mathrm{X}$ and $\mathrm{Z}$ 's location.

If $\left[\left(\mathrm{X}_{1}<\mathrm{X}_{2}<\mathrm{X}_{3}\right)\right.$ and $(\operatorname{cost}(\mathrm{XY})+\cos (\mathrm{YZ})<\cos t$

$(\mathrm{XZ})$ )]

$\mathrm{Y}$ is a common node.

4. $\mathrm{Y}$ is not a common node.

Node $\mathrm{X}$ finds common node between it and its neighbors using common node finding algorithm. Node $\mathrm{X}$ gets location information of its neighbors from Table 4. Using commonnode finding algorithm it finds the common node between two nodes as given in Table 5.

Table 5: Modified Vicinity Table of node X showing Common Node

\begin{tabular}{|c|c|c|c|c|c|}
\hline $\begin{array}{c}\text { Sender } \\
\text {-ID }\end{array}$ & $\begin{array}{c}\text { Location } \\
\text {-Info }\end{array}$ & $\begin{array}{c}\text { Min- } \\
\text { comm- } \\
\text { cost }\end{array}$ & $\begin{array}{c}\text { Common } \\
\text {-node }\end{array}$ & $\begin{array}{c}\text { Direct } \\
- \\
\text { comm } \\
\text {-cost }\end{array}$ & $\begin{array}{c}\text { Link } \\
\text {-type }\end{array}$ \\
\hline $\mathrm{Y}$ & 101,96 & 01 & - & 01 & $\mathrm{D}$ \\
\hline $\mathrm{Z}$ & 102,98 & $\begin{array}{c}5+1=0 \\
6\end{array}$ & $\mathrm{Y}$ & 08 & $\mathrm{I}$ \\
\hline $\mathrm{M}$ & 100,99 & 09 & - & 09 & $\mathrm{D}$ \\
\hline $\mathrm{N}$ & 98,94 & 08 & - & 08 & $\mathrm{D}$ \\
\hline $\mathrm{O}$ & 99,92 & $8+5=1$ & $\mathrm{~N}$ & 17 & $\mathrm{I}$ \\
& & 3 & & & \\
\hline
\end{tabular}

This information helps to find list of direct and indirect neighbors of node $\mathrm{X}$. From this list node $\mathrm{X}$ will choose the farthest direct node using farthest-direct-node finding algorithm. Farthest direct node is the node present at maximum distance in node $\mathrm{X}$ 's transmission range. The directcomm-cost of this farthest direct node is set as $\mathrm{P}_{\text {data }}(\mathrm{X})$ [12].
$\mathrm{P}_{\text {data }}(\mathrm{x})$ is the new transmission power of node $\mathrm{X}$. Every direct neighbor of node $\mathrm{X}$ is reachable within this new transmission range.

Farthest-direct-node finding algorithm ( ):

1. //Initialize record to 1 st record of vicinity table and direct-cost [ ] array stores the direct-comm-cost of nodes having link-type 'd'

2. $\mathrm{i}=0$

3. while ( record != null)

\{

if (record.link-type== 'd') then

\{

Insert record to direct-cost [i]

$\mathrm{i}++$

\}

record $\rightarrow$ record. next

\}

4. Find max (direct-cost []$, \mathrm{n}) / / \mathrm{n}$ is the length of array direct-cost [ ]

5. Find sender-ID of max (direct-cost [i] ) //sender-ID is the farthest direct node.

Some indirect neighbor nodes are also reachable within this new transmission range of node $\mathrm{X}$. Their direct-comm-cost lies within this range. The link-type field of these nodes are updated from " $\mathrm{i}$ " to "d". Node X can directly transmit to those nodes. It does not need to go through common node. For this final vicinity table updating algorithm is used. It updates the vicinity table to a final one as given in Table 6 .

Final Vicinity Table updating algorithm ( ):

1. // Initialize record to 1 st record of vicinity table

2. while ( record $!=$ null)

\{

if $($ record.link-type $==$ ' $i$ ')

\{

find record.direct-comm-cost

if ( record.direct-comm-cost $<\mathrm{P}_{\text {data }}(\mathrm{X})$ ) // node is in transmission range of node $\mathrm{X}$

link-type = 'd' // update link-type to "d" from "i"

\}

record $\rightarrow$ record. next

\} 
Table 6: Final Vicinity Table of node $X$

\begin{tabular}{|c|c|c|c|c|c|}
\hline $\begin{array}{c}\text { Sender } \\
\text {-ID }\end{array}$ & $\begin{array}{c}\text { Location } \\
\text {-Info }\end{array}$ & $\begin{array}{c}\text { Min- } \\
\text { comm- } \\
\text { cost }\end{array}$ & $\begin{array}{c}\text { Common } \\
\text {-node }\end{array}$ & $\begin{array}{c}\text { Direct } \\
- \\
\text { comm } \\
\text {-cost }\end{array}$ & $\begin{array}{c}\text { Link } \\
\text {-type }\end{array}$ \\
\hline $\mathrm{Y}$ & 101,96 & 01 & - & 01 & $\mathrm{D}$ \\
\hline $\mathrm{Z}$ & 102,98 & $\begin{array}{c}5+1=0 \\
6\end{array}$ & $\mathrm{Y}$ & 08 & $\mathrm{D}$ \\
\hline $\mathrm{M}$ & 100,99 & 09 & - & 09 & $\mathrm{D}$ \\
\hline $\mathrm{N}$ & 98,94 & 08 & - & 08 & $\mathrm{D}$ \\
\hline $\mathrm{O}$ & 99,92 & $8+5=1$ & $\mathrm{~N}$ & 17 & $\mathrm{I}$ \\
& & 3 & & & \\
\hline
\end{tabular}

The direct and indirect neighbors are find out from X's final vicinity table. This is the result of our first phase. In the same way each node only needs to broadcast the hello message once, is attained quickly and easily. An appropriate transmission power for each node is decided after they carry out first phase. A node continuously checks which neighbors it can exclude from its direct communication to conserve power. At the end of which every node in network determines its final vicinity table.

\subsection{Sleep Scheduling Phase}

At the end of link-determination phase each node is having its final vicinity table. Every node starts to respond to sleep scheduling phase depending upon the information from its final vicinity table. In this phase each node utilizes its data transmission power efficiently. The node in the farthest transmission range is considered as next hop. This node is geographically closer to the destination node. The other nodes not participating in routing under goes for sleep state. The next hop finding algorithm is stated in following algorithm.

Next hop node finding procedure ( ):

1. //Destination-location is given from vicinity table say $\left(\mathrm{X}_{1}, \mathrm{Y}_{1}\right)$

2. Initialize record to 1 st record of vicinity table while ( record != null)

\{

$$
\text { if }(\text { record.link-type }==\text { ' } d \text { ') }
$$

\{

Find location-info //say $\left(\mathrm{X}_{2}, \mathrm{Y}_{2}\right)$

$$
\begin{aligned}
& \left.d=\sqrt{\left(X_{2}-X_{1}\right)^{2}-(} Y_{2}-Y_{1}\right)^{2} / / \text { Find distance } \\
& \text { between }\left(\mathrm{X}_{2}, \mathrm{Y}_{2}\right) \text { and }\left(\mathrm{X}_{1}, \mathrm{Y}_{1}\right) \\
& \text { record } \rightarrow \text { record. next } \\
& \text { Compare the distance and find minimum distance }
\end{aligned}
$$

\}
As per the algorithm the node calculates the distance between its direct neighbors and the destination node. Then it finds for which direct node the distance is coming minimum. That node is considered as next hop. The other nodes not involving in routing are in idle mode. Energy is conserved by putting those idle mode node into sleep state. For example in Table 6 node $\mathrm{X}$ has four direct nodes $\mathrm{Y}, \mathrm{Z}, \mathrm{M}$ and $\mathrm{N}$. The distance is calculated between these direct nodes and destination node $\mathrm{D}$. The calculated distances are as given bellow:

$\mathrm{M}(100,99) \rightarrow \mathrm{D}(400,100)=300$

$\mathrm{Y}(101,96) \rightarrow \mathrm{D}(400,100)=299$

$\mathrm{N}(98,94) \rightarrow \mathrm{D}(400,100)=302$

$\mathrm{Z}(102,98) \rightarrow \mathrm{D}(400,100)=298$

Following in this way it successively follows the close geographic hops. It traverses less number of hops in energetically efficient manner to reach the destination. Using above algorithm node $\mathrm{X}$ chooses node $\mathrm{Z}$ as its next hop neighbor to forward packet to node $\mathrm{D}$. So node $\mathrm{Z}$ is in active mode, while the other nodes are in idle mode. In idle mode it consumes energy unnecessarily. So it's better to put idle mode node in sleep mode to conserve energy.

Table 7: Inform Message Format

\begin{tabular}{|l|l|l|}
\hline Source-ID & Msg-type & Target-node \\
\hline
\end{tabular}

From calculated distances node $\mathrm{Z}$ is considered as next hope node. The other nodes not involving in packet transmission are put to sleep state. Node $\mathrm{X}$ is using the inform message format as given in Table 7 .

Under the Msg-type header node X writes the message (Sleep, Time) as given in Table 8. Time specifies the duration for which the idle mode nodes are put to sleep state. Node $\mathrm{Z}$ is chosen as

Table 8: Inform Message Format

\begin{tabular}{|c|c|c|}
\hline Source-ID & Msg-type & Target-node \\
\hline $\mathrm{X}$ & Sleep, Time & $\sim \mathrm{Z}$ \\
\hline
\end{tabular}

next hop node of node $\mathrm{X}$ for packet transmission to node $\mathrm{D}$. So the sleep message is for all neighbors of node except node $Z$. It is specified under Target-node field of inform message. In the same manner node $\mathrm{Z}$ finds its next hop node to the destination. It uses sleep based approaches to idle mode nodes. Following in this way the packet successively follows the close geographic hops to reach the destination.

\section{SIMULATIONS AND RESULTS}

The performance of our proposed protocol (DBSS) is compared with that of existing (LFTC) algorithm through simulation using Qualnet 4.5 simulator. We have used three parameters such as energy consumption, average end-to-end delay and throughput for measuring performance. The performance of these algorithms has been studied (i) by varying the number of packets and (ii) by changing mobile speed. 


\subsection{By Varying Number of Packets}

Performance metrics considered for comparison are: (1) Energy consumption vs. no. of packets (2) End to end delay vs. no. of packets and (3) Throughput vs. no. of packets. Parameters considered for simulation is shown in Table 9. The number of packets to be sent through CBR traffic is varied from 100 to 1000 in numbers.

Table 9: Simulation Parameters

\begin{tabular}{|c|c|}
\hline Parameter & Value \\
\hline Terrain Co-ordinates & $1000,1000 \mathrm{~m}^{2}$ \\
\hline Simulation Time & $30 \mathrm{~min}$ \\
\hline Maximum nodes & 8 \\
\hline Routing protocol & AODV \\
\hline Traffic & CBR \\
\hline Item size & $512 \mathrm{bytes}$ \\
\hline Interval time of item sending & $1 \mathrm{sec}$ \\
\hline
\end{tabular}

The graph for energy consumption vs. no. of packets is shown in Figure 2. It is observed from the graph that the energy consumption in our proposed scheme is lesser that of the existing (LFTC). This is because in proposed scheme for

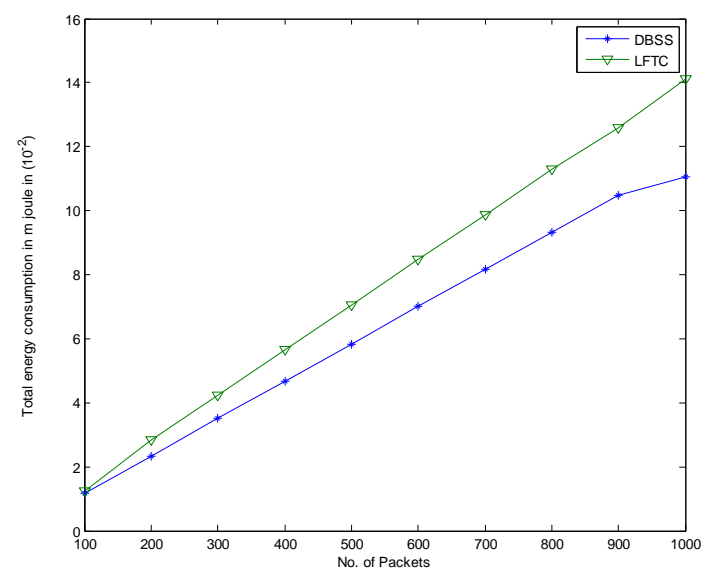

Figure 2: Total energy consumption vs. no. of packets

transmission of packet we are taking the farthest direct node coming in transmission range of a node as next hop. Here transmission is done through less number of hops. So the total energy consumption is less as less number of nodes is taking part in packet transmission as compare to existing approach.

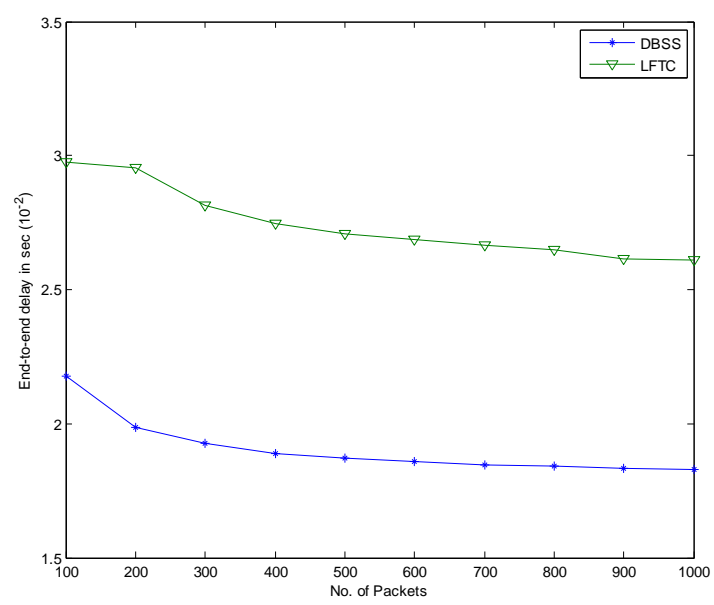

Figure 3: average end-to-end delay vs. No. of packets

The comparison for end-to-end delay in between DBSS and LFTC protocol is shown in Figure 3. It is observed from the figure that the end to end delay in our proposed scheme is lesser than that of existing approach. This is because in proposed scheme as less number of nodes are taking part in packet transmission, so the packet needs to travel less hops, resulting the average end-to-end delay is minimized.

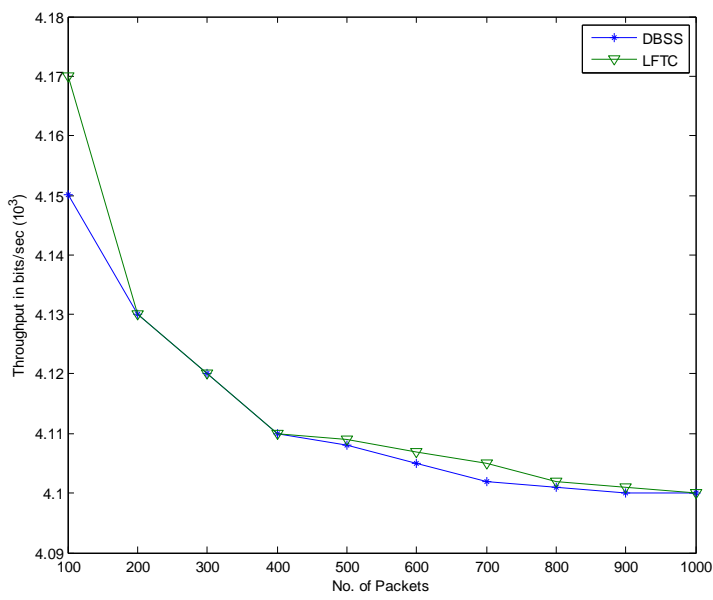

Figure 4: Throughput vs. No. of packets

The graph for throughput vs. no. of packets is plotted in Figure 4. It is observed from the figure that the throughput in our proposed scheme is lesser than that of the existing scheme for packet number 100 to 900 . From 1000 packets both the proposed and existing scheme gives same throughput.

\subsection{By Varying Mobile speed}

In this simulation speed of node is varied by keeping number of packets constant at 1000 . Performance metrics considered for comparison are: (1) Energy consumption vs. maximum speed (2) End to end delay vs. maximum speed and (3) Throughput vs. maximum speed. Parameters considered for simulation is shown in Table 10. 
Table 10: Simulation Parameters

\begin{tabular}{|c|c|}
\hline Parameter & Value \\
\hline Terrain Co-ordinates & $1000,1000 \mathrm{~m}^{2}$ \\
\hline Simulation Time & $30 \mathrm{~min}$ \\
\hline Maximum nodes & 8 \\
\hline Routing protocol & AODV \\
\hline Traffic & CBR \\
\hline Item size & 512 bytes \\
\hline No. of Packets & 1000 \\
\hline
\end{tabular}

In the mobile condition of the node the comparison is studied for the energy consumption between DBSS and LFTC protocol.

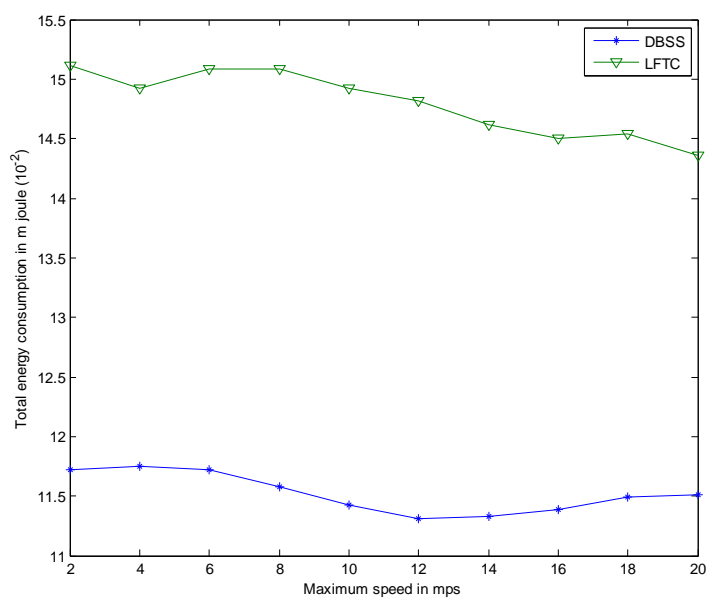

Figure 5: Total energy consumption vs. Maximum speed

It is observed from Figure 5 that the energy consumption in our proposed scheme is lesser that of the existing (LFTC). This is because in proposed scheme for transmission of packet we are taking the farthest direct node coming in transmission range of a node as next hop, so transmission is done through less number of hops, so the total energy consumption is less as less number of nodes are taking part in packet transmission as compare to existing approach.

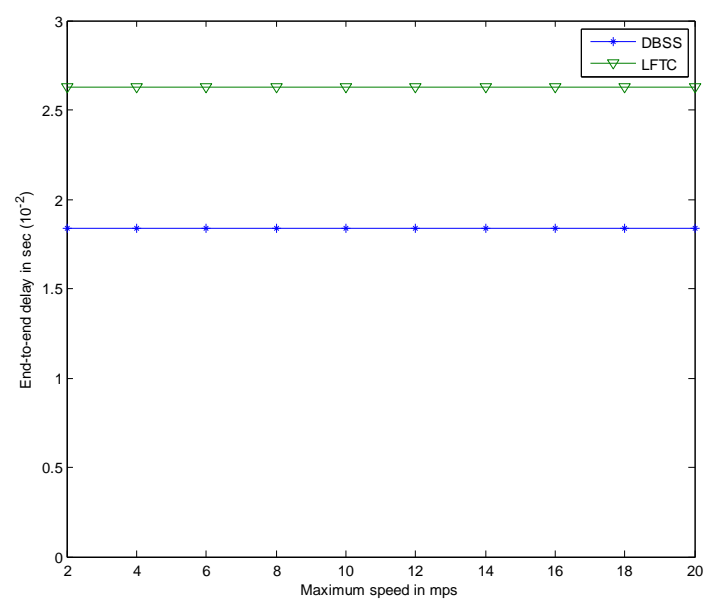

Figure 6: Average end-to-end delay Vs. Maximum speed

The graph for end to end delay vs. maximum speed is shown in Figure 6. It is observed from the graph that the end to end delay in our proposed scheme is lesser than that of existing approach. This is because in proposed scheme as less number of nodes are taking part in packet transmission, so the packet needs to travel less hops, resulting the average end-to-end delay is minimized.

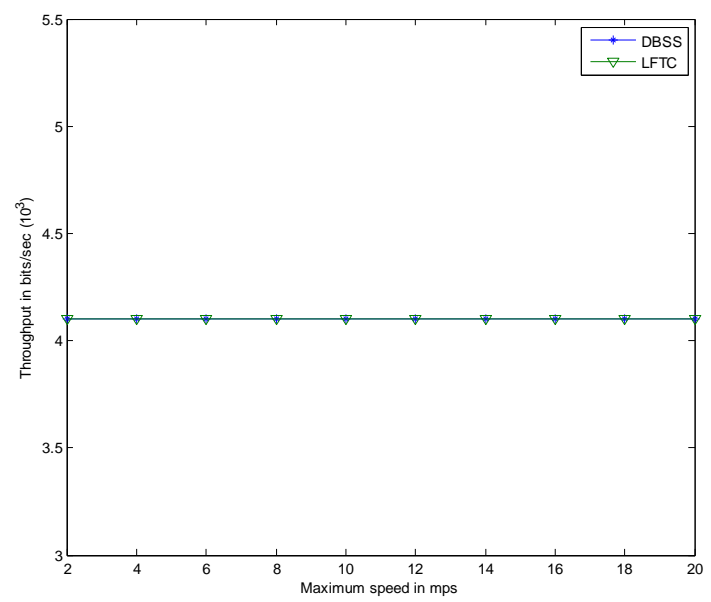

Figure 7: Throughput vs. Maximum speed

As per the Figure 7 the throughput achieved in DBSS is almost same as that of LFTC protocol for 1000 packets.

\section{CONCLUSIONS}

In this paper, we proposed a two-phase DBSS protocol which deals with topology control and provides the mechanism to reduce overall energy consumption in networks. It discusses how topology of deployment of nodes plays an important role regarding energy conservation. In DBSS protocol it takes farthest node in its transmission range for routing. That node is geographically closer to the destination. The number of nodes in packet transferring is less. Energy conservation is done by utilizing energy of small set of nodes. Sleep based approaches for the other idle mode node minimizes energy 
consumption of the network. The proposed DBSS protocol is compared with LFTC using Qualnet 4.5 simulator. Simulation results proved that our DBSS protocol has a good energy conservation performance. DBSS also performs better in context of average end-to-end delay without much affecting the throughput.

\section{ACKNOWLEDGEMENTS}

This research was supported by project grant Vide No: 13(1)2008-CC\&BT of Ministry of Communication \& Information Technology, Dept of IT, Government of India, titled "Energy Aware Protocol for Wireless Networks" and being carried out at department of Computer Science and Engineering, NIT Rourkela.

\section{REFERENCES}

[1] S. Rout, A.K. Turuk and B. D. Sahoo, "Energy Efficiency in Wireless Network: Through Alternate path Routing", in proceedings of Fourth Innovative conference on Embedded Systems, Mobile Communication and Computing, July 2009, pp.-3-8.

[2] S. Rout, A. K. Turuk and B. D. Sahoo, "Energy Efficiency in Wireless Ad hoc Network using Clustering", in proceedings on $12^{\text {th }}$ International Conference on Information Technology, December 2009, pp.-223-226.

[3] S. K. Sarkar, T. G. Basavaraju, and C. Puttamadappa, "Ad Hoc Mobile Wireless Networks", Auerbach Publications, 2007

[4] P. Santi, "Topology Control in Wireless Ad Hoc and Sensor Networks", John Wiley \$ Sons, Ltd., 2006.

[5] S.-L. Wu and Y.-C. Tseng. "Wireless Ad Hoc Networking". Auerbach Publications, 2007.

[6] M. Tarique, K. E. Tepe, and M. Naserian. "Energy Saving Dynamic Source Routing for Ad Hoc Wireless Networks". Proceedings in Third International symposium on Modeling and Optimization in Mobile Ad Hoc, and Wireless Networks, Canada, pages 305-310, April 2005.

[7] E. M. Royer and C. K. Toh. "A Review of Current Routing Protocol for Ad Hoc Mobile Wireless Networks". Proceedings in IEEE Personal Communication Magazine, 6(2): 46-55, April 1999.
[8] N. Gupta and S. R. Das. "Energy-Aware On-Demand Routing for Mobile Ad Hoc Networks". Proceedings of the4th International Workshop on Distributed Computing, Mobile and Wireless Computing, Lecture notes in computer Science, 2571: 164-173, January 2002.

[9] A. Avudainayagam, W. Lou, and Y. Fang. "DEAR: A Device and Energy Aware Routing protocol for Heterogeneous Ad Hoc Networks". Proceedings in Academic press Journals of Parallel and Distributed Computing, 63(2): 228-236, February 2003.

[10] B. Chen, K. Jamieson, H. Balakrishnan, and R. Morris. "Span: An Energy-Efficient Coordination Algorithm for Topology Maintenance in Ad Hoc Wireless Networks". Proceedings in Academic Publishers of Wireless Networks, 8(5): 481-494, September 2002.

[11] S. Banerjee and A. Misra. "XTC: A Practical Topology Control Algorithm for Ad-Hoc Networks". Proceedings in $18^{\text {th }}$ International Symposium on Parallel and Distributed Processing, Switzerland, 216-223, April 2004.

[12] J.-P. Sheu, S.-C. Tu, and C.-H. Hsu. "Location-Free Topology Control Protocol in Wireless Ad Hoc Networks". Computer Communications, 31(14): 34103419, 2008 .

[13] X. Shen and L. Meng, "Energy-Aware Routing Protocol in Fading Channel for Mobile Ad Hoc Networks", Proceedings in IEEE International conference on Information Theory and Information Security, 871-875, December 2010.

[14] D. Zappala. "Alternate Path Routing for Multicast". Proceedings in IEEE/ACM Transactions on Networking, 12(1): 30-43, February 2004.

[15] S.-L. Wu, Y.-C. Tseng, and J.-P. Sheu. "Intelligent Medium Access for Mobile Ad Hoc Networks with Busy Tones and Power Control". IEEE Journal on Selected Areas in Communications, 18(9): 1647-1657, 2000.

[16] Y. Xu, J. Heidemann, and D. Estrin. "GeographyInformed Energy Conservation for Ad Hoc Routing". Proceedings of the 7th annual International Conference on Mobile Computing and Networking, Rome, Italy, 7084 , July

2001 